\title{
Arbitrary Solution of the Schrödinger Equation Interacting with the Superposition of Hulthen with Spin- orbit Plus Adjusted Coulomb Potential Model
}

\author{
E. A. Thompson, I. O. Akpan, and C. A. Thompson
}

\begin{abstract}
In this study, we solve the non-relativistic radial part of the Schrödinger wave equation for the superposition of the Hulthen with spin-orbit plus adjusted Coulomb potential (SHSC) potential using the Nikiforov-Uvarov (NU) method for arbitrary states. The Hulthen with spin-orbit plus adjusted Coulomb (SHSC) potential is the simplest potential field for a nuclear system and has been used to obtain the single particle energy spectrum for both nucleon species orbiting a closed nuclear core. We also obtained in this study the corresponding single particle normalized wave function expressed in terms of the Jacobi polynomial. Besides, we obtained two special cases of the energy spectra for the SHSC potential.
\end{abstract}

Keywords - Schrodinger equation, spin-orbit interaction, Hulthen potential, Coulomb potential, energy eigenvalue, eigenfunction.

\section{INTRODUCTION}

There has been a concerted effort to obtain the exact bound - state solution of the wave equation, within relativistic and non-relativistic quantum mechanics. A plethora of problems involving the multidimensional Schrodinger equation have been solved over the past decades. Various analytical methods have been deployed to find a solution to the non-relativistic Schrodinger wave equation. Common amongst these methods are the asymptotic iteration method [1], supersymmetric shape invariance approach [2], exact or proper quantization rule [3], factorization method [4], 1/N shifted expansion method [5] and NikiforovUvarov method [6]. In recent times, the focus has been on combining two or more potentials to model a more compact effective potential that can be applicable to real physical systems. For instance, Agboola [7] obtained the eigenvalues and the corresponding eigen function for Hulthen potential plus the Mie Ring Shape potential, Alberg and Wilets [8] combined Coulomb and Harmonic Oscillator potentials to find the exact solution of the Schrodinger equation. Worthy of note is the work of Niknam et al [9], which provided the solution to the Schrodinger wave equation by combing Woods-Saxon with spin-orbit interaction and Coulomb potentials as an effective potential.

In the present study, we constructed an effective potential which we named SHSC potential, which models to an acceptable extent the nuclear potential. SHSC is an acronym for the superposition of Hulthen with Spin-orbit interaction and adjusted Coulomb potential.

The Hulthen potential is one of the reputable short range potentials which can easily approximate the Woods-Saxon potential [10] and exhibits the characteristics of the generalized Coulomb potential for small values of $\mathrm{r}$ and decreases exponentially for large values of $\mathrm{r}$. The Hulthen potential has been used extensively in many areas of physics including nuclear, atomic, particle and solid state physics. In this work, we present Hulthen potential as a mean filed nuclear potential. Here it is used to describe the nucleonnucleon interaction as well as the interaction between a single nucleon with the nuclear core. However, extensive studies has been carried with Hulthen potential within the relativistic and non-relativistic quantum mechanics respectively [11]-[15].

A more realistic potential for the single particle nuclear system can be obtained by combining the mean - field potential (Hulthen as in our case) [16], spin - orbit interaction [17] and the Coulomb potential (considering the interactions between protons) [18]. This combination along with the centrifugal term forms the effective potential for the nuclear system. The SHSC potential is applied to find the solution to Schrodinger wave equation to obtain the single particle energy spectrum and the corresponding wave function for the nuclear system.

Submitted on November 02, 2021

Published on December 23, 2021.

E. A. Thompson, University of Calabar, Nigeria.

(e-mail: edyy7000@yahoo.com)

I. O. Akpan, University of Calabar, Nigeria.

(e-mail: Itaeyenakpan ${ }^{\circledR}$ yahoo.com)

C. A. Thompson, University of Calabar, Nigeria.

(e-mail: cherishthompson ${ }^{\circledR}$ gmail.com) 


\section{REVIEW OF THE NU METHOD}

We take a brief overview of the NU method in this section; details can be obtained from ref. [18]. This method involves solving a hyper-geometric type second order differential equation:

$$
\psi^{\|}(\mathrm{z})+\frac{\tilde{\tau}(\mathrm{z})}{\sigma(\mathrm{z})} \psi^{\|}(\mathrm{z})+\frac{\tilde{\sigma}(\mathrm{z})}{\sigma^{2}(\mathrm{z})} \psi(\mathrm{z})=0
$$

where $\sigma(z)$ and $\tilde{\sigma}(z)$ are second order polynomials. $\tilde{\tau}(z)$ is a order degree polynomial [19]. A possible solution of Eq. (1) is proposed as:

$$
\psi(\mathrm{z})=\phi(\mathrm{z}) \mathrm{y}(\mathrm{z})
$$

which results in a hyper geometric - type equation of the form:

$$
\sigma(z) y^{\| \prime}(z)+\tau(z) y^{\prime}(z)+\lambda y(z)=0
$$

Part of the solution of Eq.(1) given in Eq. (2) as $\phi(z)$ is the solution to another differential equation of the form given by:

$$
\sigma(\mathrm{z}) \phi^{\prime}(\mathrm{z})-\pi(\mathrm{z}) \phi(\mathrm{z})=0
$$

where

$$
\tau(\mathrm{z})=\tau(\mathrm{z})+2 \pi(\mathrm{z})
$$

and the parameter $\lambda$ in Eq. (3) is defined as:

$$
\lambda=\lambda_{\mathrm{n}}=-\mathrm{n} \tau^{\prime}(\mathrm{z})+\frac{\mathrm{n}(\mathrm{n}-1)}{2} \sigma^{\|}(\mathrm{z})=0(\mathrm{n}=0,1,2,3, \ldots)
$$

The term $\tau(\mathrm{z})$ is a polynomial, and to obtain a proper solution for the hyper-geometric type differential equation, its first derivative $\tau^{\prime}(\mathrm{z})$ must be negative. The function $\mathrm{y}(\mathrm{z})$ as stated in Eq. (2) is the hyper geometric - type wave function obtained using the Rodrigues relation:

$$
\mathrm{y}_{\mathrm{n}}(\mathrm{z})=\frac{\mathrm{B}_{\mathrm{n}}(\mathrm{z}) \mathrm{d}^{\mathrm{n}}}{\rho(\mathrm{z}) \mathrm{dz^{ \textrm {n } }}}\left[\sigma^{\mathrm{n}}(\mathrm{z}) \rho(\mathrm{z})\right]
$$

where $B_{n}$ is related to the normalization constant, and $\rho(z)$ is defined as:

$$
\mathrm{y}_{\mathrm{n}}(\mathrm{z})=\frac{\mathrm{B}_{\mathrm{n}}(\mathrm{z}) \mathrm{d}^{\mathrm{n}}}{\rho(\mathrm{z}) \mathrm{dz}}\left[\mathrm{z}^{\mathrm{n}}(\mathrm{z}) \rho(\mathrm{z})\right]
$$

Also the function $\pi(\mathrm{z})$ is a first order polynomial is defined as:

$$
\pi(\mathrm{z})=\frac{\sigma^{\prime}(\mathrm{z})-\tilde{\tau}(\mathrm{z})}{2} \pm \sqrt{\left(\frac{\sigma^{\prime}(\mathrm{z})-\tilde{\tau}(\mathrm{z})}{2}\right)^{2}-\tilde{\sigma}(\mathrm{z})+\mathrm{k} \sigma(\mathrm{z})}
$$

$\mathrm{K}$ in Eq. (9) is related to Eq. (6) and the first derivative of $\pi^{\prime}(z)$ as thus:

$$
\lambda=\mathrm{k}+\pi^{\prime}(\mathrm{z})
$$

The value of $\mathrm{k}$ is obtained by equating the discriminant of the quadratic expression under the square root sign in Eq. (9) to zero. By solving Eq. (6) and Eq. (10), we derive the energy eigenvalue equation. 


\section{SOLUTION OF SCHRODINGER EQUATION FOR SHSC}

The single particle energy spectrum $\mathrm{E}_{\mathrm{n} \ell \mathrm{j}}$ for nuclei is obtained by solving the radial component of the 3dimensional Schrodinger wave equation given as:

$$
\frac{\mathrm{d}^{2} \mathrm{R}(\mathrm{r})}{\mathrm{dr}^{2}}+\frac{2}{\mathrm{r}} \frac{\mathrm{dR}(\mathrm{r})}{\mathrm{dr}}+\frac{2 \mu}{\hbar^{2}}\left[\mathrm{E}_{\mathrm{n} \ell \mathrm{j}}-\mathrm{V}_{\mathrm{eff}}(\mathrm{r})\right] \mathrm{R}(\mathrm{r})=0
$$

where $\mu$ is the reduced mass of a single nucleon, $E_{n \ell j}$ is the energy spectra, $\hbar$ is the Planck's constant, $n$, $\ell$ and $\mathrm{j}$ are the principal, orbital and total momentum quantum number respectively. Let define a new variable be defined as:

$$
\mathrm{z}=\mathrm{e}^{-2 \alpha \mathrm{r}}
$$

The derivatives of the function $\mathrm{R}(\mathrm{r})$ in Eq. (11) are obtained in terms of the new variable z. Equation (11) is transformed and restated in terms of $\mathrm{z}$, as $\mathrm{R}(\mathrm{r})$ is redefined as $\psi(\mathrm{z})$. The attempt is to provide an approximate but not exact solution to the Schrodinger equation for the effective potential employed in the present work.

Using the variable as defined by Eq. (12), Eq. (11) is transformed and the Schrodinger equation becomes as below:

$$
\begin{aligned}
& \frac{\mathrm{d}^{2} \psi(\mathrm{z})}{\mathrm{dz}^{2}}+\frac{1}{\mathrm{z}} \frac{\mathrm{d} \psi(\mathrm{z})}{\mathrm{dz}}+\frac{1}{\alpha^{2} \mathrm{z}^{2}}\left[\frac{2 \mu \mathrm{E}}{\hbar^{2}}-\frac{2 \mu}{\hbar^{2}}\left(\mathrm{~V}_{\text {eff }}\right)\right]=0 \\
& \frac{\mathrm{d}^{2} \psi(\mathrm{z})}{\mathrm{dz}^{2}}+\frac{1}{\mathrm{z}} \frac{\mathrm{d} \psi(\mathrm{z})}{\mathrm{dz}}+\frac{1}{\alpha^{2} \mathrm{z}^{2}}\left[\frac{2 \mu \mathrm{E}}{\hbar^{2}}-\frac{2 \mu}{\hbar^{2}}\left(\mathrm{~V}_{\text {eff }}\right)\right]=0
\end{aligned}
$$

The effective potential $\mathrm{V}_{\text {eff }}$ in Eq. (13) is the SHSC potential which is a superposition of Hulthen, spinorbit interaction, adjusted Coulomb and the centrifugal term. The Hulthen potential is as defined by Eq. (14).

$$
\mathrm{V}_{\mathrm{H}}(\mathrm{r})=-\frac{\mathrm{V}_{\mathrm{o}} \alpha \mathrm{e}^{-\alpha \mathrm{r}}}{1-\mathrm{e}^{-\alpha \mathrm{r}}}
$$

where $V_{o}$ in Eq. (14) is the potential strength of the Hulthen potential.

The adjusted Coulomb potential is given as:

$$
\mathrm{V}_{\mathrm{c}}(\mathrm{r})=\frac{\mathrm{e}^{2}}{\mathrm{R}_{\mathrm{o}}}\left[3-\left(\frac{\mathrm{r}}{\mathrm{R}_{\mathrm{o}}}\right)^{2}\right]
$$

where $e$ is the charge, $R_{o}$ the nuclei radius and $r$ is the range of this of the position of a particle from the core.

The spin-orbit interaction is given as:

$$
\mathrm{V}_{\mathrm{LS}}(\mathrm{r})=\frac{1}{2} \mathrm{~V}_{\mathrm{LS}}(0)\left(\frac{\mathrm{r}_{\mathrm{o}}}{\hbar}\right)^{2} \frac{1}{\mathrm{r}}\left[\frac{\mathrm{dV}_{\mathrm{H}}(\mathrm{r})}{\mathrm{dr}}\right] \overrightarrow{\mathrm{L} . S}
$$

where $\overrightarrow{\mathrm{L}} \cdot \overrightarrow{\mathrm{S}}=\frac{\hbar^{2}}{2}\left(\mathrm{j}(\mathrm{j}+1)-\ell(\ell+1)-\frac{3}{4}\right)$

The centrifugal term of the Schrodinger wave equation is given as:

$$
\mathrm{V}_{\mathrm{cf}}=\frac{\hbar^{2}}{2 \mu} \frac{\ell(\ell+1)}{\mathrm{r}^{2}}
$$

The SHSC potential $V_{\text {eff }}$ for the nuclei system is obtained by combining Eq. (14), Eq. (15), Eq.(16) and Eq.(17) as stated in Eq.(18):

$$
\mathrm{V}_{\text {eff }}=-\frac{\mathrm{V}_{\mathrm{o}} \mathrm{e}^{-\alpha \mathrm{r}}}{1-\mathrm{e}^{-\alpha \mathrm{r}}}-\mathrm{V}_{\mathrm{LS}}(0) \mathrm{r}_{\mathrm{o}}^{2} \frac{1}{\mathrm{r}} \frac{\alpha \mathrm{e}^{-\alpha \mathrm{r}}}{\left(1-\mathrm{e}^{-\alpha \mathrm{r}}\right)^{2}} \overrightarrow{\mathrm{L}} \cdot \overrightarrow{\mathrm{S}}-\frac{3 \mathrm{e}^{2}}{\pi \varepsilon_{0} \mathrm{R}_{\mathrm{o}}}+\frac{\mathrm{e}^{2} \mathrm{r}^{2}}{\pi \varepsilon_{0} \mathrm{R}_{\mathrm{o}}^{3}}+\frac{\hbar^{2}}{2 \mu} \frac{\ell(\ell+1)}{\mathrm{r}^{2}}
$$


By the application of the Greene-Aldrich approximations scheme [20], [21], [22] the centrifugal barrier is handled. This approximation scheme is valid for $\alpha \ll 1$. And it is of the following forms:

$$
\begin{gathered}
\frac{1}{\mathrm{r}^{2}} \approx \frac{4 \alpha^{2} \mathrm{e}^{-2 \alpha \mathrm{r}}}{\left(1-\mathrm{e}^{-2 \alpha \mathrm{r}}\right)^{2}} \\
\frac{1}{\mathrm{r}^{2}} \approx \frac{\alpha^{2}}{\left(1-\mathrm{e}^{-2 \alpha \mathrm{r}}\right)^{2}}
\end{gathered}
$$

Substituting Eq. (18), Eq. (19) and Eq. (20) into Eq. (13), the Schrodinger equation now reads:

$$
\frac{\mathrm{d}^{2} \psi(\mathrm{z})}{\mathrm{dz}^{2}}+\frac{1}{\mathrm{z}} \frac{\mathrm{d} \psi(\mathrm{z})}{\mathrm{dz}}+\frac{1}{\mathrm{z}^{2}(1-\mathrm{z})^{2}}\left[\begin{array}{l}
\frac{2 \mu \mathrm{E}}{\hbar^{2} \alpha^{2}}(1-\mathrm{z})^{2}+\mathrm{V}_{\mathrm{o}} \frac{2 \mu}{\hbar^{2} \alpha^{2}} \mathrm{z}(1-\mathrm{z}) \\
+\mathrm{V}_{\mathrm{LS}}(0) \frac{2 \mu \mathrm{r}_{\mathrm{o}}^{2}}{\hbar^{2}}\left(\frac{\mathrm{z}}{1-\mathrm{z}}\right)\left(\mathrm{j}(\mathrm{j}+1)-\ell(\ell+1)-\frac{3}{4}\right) \\
-\frac{6 \mu \mathrm{e}^{2}}{\mathrm{R}_{\mathrm{o}} \pi \varepsilon_{0} \alpha^{2} \hbar^{2}}(1-\mathrm{z})^{2}+\frac{2 \mu \mathrm{e}^{2}}{\hbar^{2} \pi \varepsilon_{0} \alpha^{4} \mathrm{R}_{\mathrm{o}}^{3}}(1-\mathrm{z})^{4} \\
-\ell(\ell+1) \mathrm{z}
\end{array}\right] \psi(\mathrm{z})=0
$$

By applying the principles of truncation as suggested by [23] to the higher powers of $\mathrm{z}$ beyond the second degree, Eq. (21) is transformed to the hyper geometric-type second order differentiate equation solvable by the NU method.

$$
\frac{\mathrm{d}^{2} \psi(\mathrm{z})}{\mathrm{dz}}+\frac{(1-\mathrm{z})}{\mathrm{z}(1-\mathrm{z})} \frac{\mathrm{d} \psi(\mathrm{z})}{\mathrm{dz}}+\frac{1}{\mathrm{z}^{2}(1-\mathrm{z})^{2}}\left[\begin{array}{l}
-\frac{2 \mu \mathrm{E}}{\hbar^{2} \alpha^{2}} \mathrm{z}^{2}+\mathrm{V}_{\mathrm{LS}}(0) \frac{2 \mu \mathrm{r}_{\mathrm{o}}^{2}}{\hbar^{2}}\left(\mathrm{j}(\mathrm{j}+1)-\ell(\ell+1)-\frac{3}{4}\right) \mathrm{z}^{2} \\
-\frac{6 \mu \mathrm{e}^{2}}{\mathrm{R}_{\mathrm{o}} \pi \varepsilon_{0} \alpha^{2} \hbar^{2}} \mathrm{z}^{2}+6 \frac{2 \mu \mathrm{e}^{2}}{\hbar^{2} \pi \varepsilon_{0} \alpha^{4} \mathrm{R}_{\mathrm{o}}^{3}} \mathrm{z}^{2}-\mathrm{V}_{\mathrm{o}} \frac{2 \mu}{\hbar^{2} \alpha} \mathrm{z}^{2} \\
+2 \frac{2 \mu \mathrm{E}}{\hbar^{2} \alpha^{2}} \mathrm{z}+\mathrm{V}_{\mathrm{LS}}(0) \frac{2 \mu \mathrm{r}_{\mathrm{o}}^{2}}{\hbar^{2}}\left(\mathrm{j}(\mathrm{j}+1)-\ell(\ell+1)-\frac{3}{4}\right) \mathrm{z} \\
+2 \frac{6 \mu \mathrm{e}^{2}}{\mathrm{R}_{\mathrm{o}} \pi \varepsilon_{0} \alpha^{2} \hbar^{2}} \mathrm{z}-4 \frac{2 \mu \mathrm{e}^{2}}{\hbar^{2} \pi \varepsilon_{0} \alpha^{4} \mathrm{R}_{\mathrm{o}}^{3}} \mathrm{z}-\ell(\ell+1) \mathrm{z} \\
+\mathrm{V}_{\mathrm{o}} \frac{2 \mu}{\hbar^{2} \alpha} \mathrm{z}-\frac{2 \mu \mathrm{E}}{\hbar^{2} \alpha^{2}}-\frac{6 \mu \mathrm{e}^{2}}{\mathrm{R}_{\mathrm{o}} \pi \varepsilon_{0} \alpha^{2} \hbar^{2}}+\frac{2 \mu \mathrm{e}^{2}}{\hbar^{2} \pi \varepsilon_{0} \alpha^{4} \mathrm{R}_{\mathrm{o}}^{3}}
\end{array}\right] \psi
$$

where

$$
\left.\begin{array}{c}
\Gamma=\left\{\begin{array}{l}
-\frac{2 \mu \mathrm{E}}{\hbar^{2} \alpha^{2}}+\mathrm{V}_{\mathrm{LS}}(0) \frac{2 \mu \mathrm{r}_{\mathrm{o}}^{2}}{\hbar^{2}}\left(\mathrm{j}(\mathrm{j}+1)-\ell(\ell+1)-\frac{3}{4}\right) \\
-\frac{6 \mu \mathrm{e}^{2}}{\mathrm{R}_{\mathrm{o}} \pi \varepsilon_{0} \alpha^{2} \hbar^{2}}+6 \frac{2 \mu \mathrm{e}^{2}}{\hbar^{2} \pi \varepsilon_{0} \alpha^{4} \mathrm{R}_{\mathrm{o}}^{3}}-\mathrm{V}_{\mathrm{o}} \frac{2 \mu}{\hbar^{2} \alpha}
\end{array}\right\} \\
\Sigma=\left\{\begin{array}{l}
2 \frac{2 \mu \mathrm{E}}{\hbar^{2} \alpha^{2}}+\mathrm{V}_{\mathrm{LS}}(0) \frac{2 \mu \mathrm{r}_{\mathrm{o}}^{2}}{\hbar^{2}}\left(\mathrm{j}(\mathrm{j}+1)-\ell(\ell+1)-\frac{3}{4}\right) \\
+2 \frac{6 \mu \mathrm{e}^{2}}{\mathrm{R}_{\mathrm{o}} \pi \varepsilon_{0} \alpha^{2} \hbar^{2}}-4 \frac{2 \mu \mathrm{e}^{2}}{\hbar^{2} \pi \varepsilon_{0} \alpha^{4} \mathrm{R}_{\mathrm{o}}^{3}}+\mathrm{V}_{\mathrm{o}} \frac{2 \mu}{\hbar^{2} \alpha}-\ell(\ell+1)
\end{array}\right\} \\
-\mathbb{Q}=\frac{2 \mu \mathrm{E}}{\hbar^{2} \alpha^{2}}+\frac{6 \mu \mathrm{e}^{2}}{\mathrm{R}_{\mathrm{o}} \pi \varepsilon_{0} \alpha^{2} \hbar^{2}}-\frac{2 \mu \mathrm{e}^{2}}{\hbar^{2} \pi \varepsilon_{0} \alpha^{4} \mathrm{R}_{\mathrm{o}}^{3}}
\end{array}\right\}
$$

Putting Eq. (23), Eq. (24) and Eq. (25) into Eq. (22), we have a second differential equation of hypergeometric type Eq. (26):

$$
\frac{\mathrm{d}^{2} \psi(\mathrm{z})}{\mathrm{dz}^{2}}+\frac{(1-\mathrm{z})}{\mathrm{z}(1-\mathrm{z})} \frac{\mathrm{d} \psi(\mathrm{z})}{\mathrm{dz}}+\frac{1}{\mathrm{z}^{2}(1-\mathrm{z})^{2}}\left[\Gamma \mathrm{z}^{2}+\Sigma \mathrm{z}-\mathbb{Q}\right] \psi(\mathrm{z})=0
$$

Comparing Eq. (26) and Eq. (1), the relevant polynomials can be expressed as thus: 


$$
\begin{aligned}
& \tilde{\tau}(\mathrm{z})=1-\mathrm{z}, \quad \sigma(\mathrm{z})=\mathrm{z}(1-\mathrm{z}), \quad \sigma^{2}(\mathrm{z})=\mathrm{z}^{2}(1-\mathrm{z})^{2} \\
& \sigma(\mathrm{z})=\Gamma \mathrm{z}^{2}+\Sigma \mathrm{z}-\mathbb{Q}
\end{aligned}
$$

Inserting the polynomials as defined by Eq. (27) into Eq. (9), we have:

$$
\pi(\mathrm{z})=\frac{-\mathrm{z}}{2} \pm \sqrt{(\gamma-\mathrm{K}) \mathrm{z}^{2}+(\Sigma+\mathrm{K}) \mathrm{z}+\mathbb{Q}}
$$

where

$$
\gamma=\frac{1}{4}+\Gamma
$$

As required by the NU method the discriminant of the quadratic expression under the square root sign in Eq. (28) is equated to zero, this result in another quadratic equation in terms of K. solving the resultant quadratic equation for $\mathrm{K}$, we have possible solutions as:

$$
\mathrm{K}=-(\Sigma+2 \mathbb{Q}) \pm 2 \sqrt{\mathbb{Q}} \sqrt{\gamma+\Sigma+\mathbb{Q}}
$$

From Eq. (30), substituting the negative solution of $\mathrm{K}$ into Eq.(28), we have:

$$
\pi(\mathrm{z})=\frac{-\mathrm{Z}}{2} \pm(\sqrt{\mathbb{Q}}+\sqrt{\gamma+\Sigma+\mathbb{Q}}) \mathrm{z}-\sqrt{\mathbb{Q}}
$$

Upon inserting Eq. (31) into Eq. (5) another required polynomial is derived:

$$
\tau(\mathrm{z})=1-2 \mathrm{z}-2(\sqrt{\mathbb{Q}}+\sqrt{\gamma+\Sigma+\mathbb{Q}}) \mathrm{z}-2 \sqrt{\mathbb{Q}}
$$

Differentiating $\tau(\mathrm{z})$ as expressed in Eq. (32) with respect to $\mathrm{z}$ we have:

$$
\tau^{\mid}(\mathrm{z})=-2-2 \sqrt{\mathbb{Q}}-2 \sqrt{\gamma+\Sigma+\mathbb{Q}}
$$

Taking the first derivative of Eq. (31) and using Eq. (30), Eq. (33) as well as Eq. (27), $\lambda$ and $\lambda_{\mathrm{n}}$ are obtained explicitly as:

$$
\begin{gathered}
\lambda=\frac{-1}{2}-\sqrt{\mathbb{Q}}-\sqrt{\gamma+\Sigma+\mathbb{Q}}-\Sigma-2 \mathbb{Q}-2 \sqrt{\mathbb{Q}} \sqrt{\gamma+\Sigma+\mathbb{Q}} \\
\lambda_{\mathrm{n}}=\mathrm{n}^{2}+\mathrm{n}+2 \mathrm{n} \sqrt{\mathbb{Q}}+2 \mathrm{n} \sqrt{\gamma+\Sigma+\mathbb{Q}}
\end{gathered}
$$

By equating Eq. (34) to Eq. (35), we solve for the term $\mathbb{Q}$ :

$$
\mathbb{Q}=\left[\frac{-1 / 2\left[(\mathrm{n}+1 / 2+\sqrt{\chi})^{2}+\frac{1}{4}+\alpha\right]}{(\mathrm{n}+1 / 2+\sqrt{\chi})}\right]^{2}
$$

where

$$
\begin{aligned}
& \chi=\gamma+\Sigma+\mathbb{Q} \\
& \alpha=-\frac{1}{4}-\gamma+\mathbb{Q}
\end{aligned}
$$

Reintroducing the Eq. (23), Eq. (24) and Eq. (25) into Eq. (37) and Eq. (36), we obtain the single particle energy spectrum for the SHSC potential in terms of the principal $n$, orbital $\ell$ and angular momentum $j$ quantum numbers: 


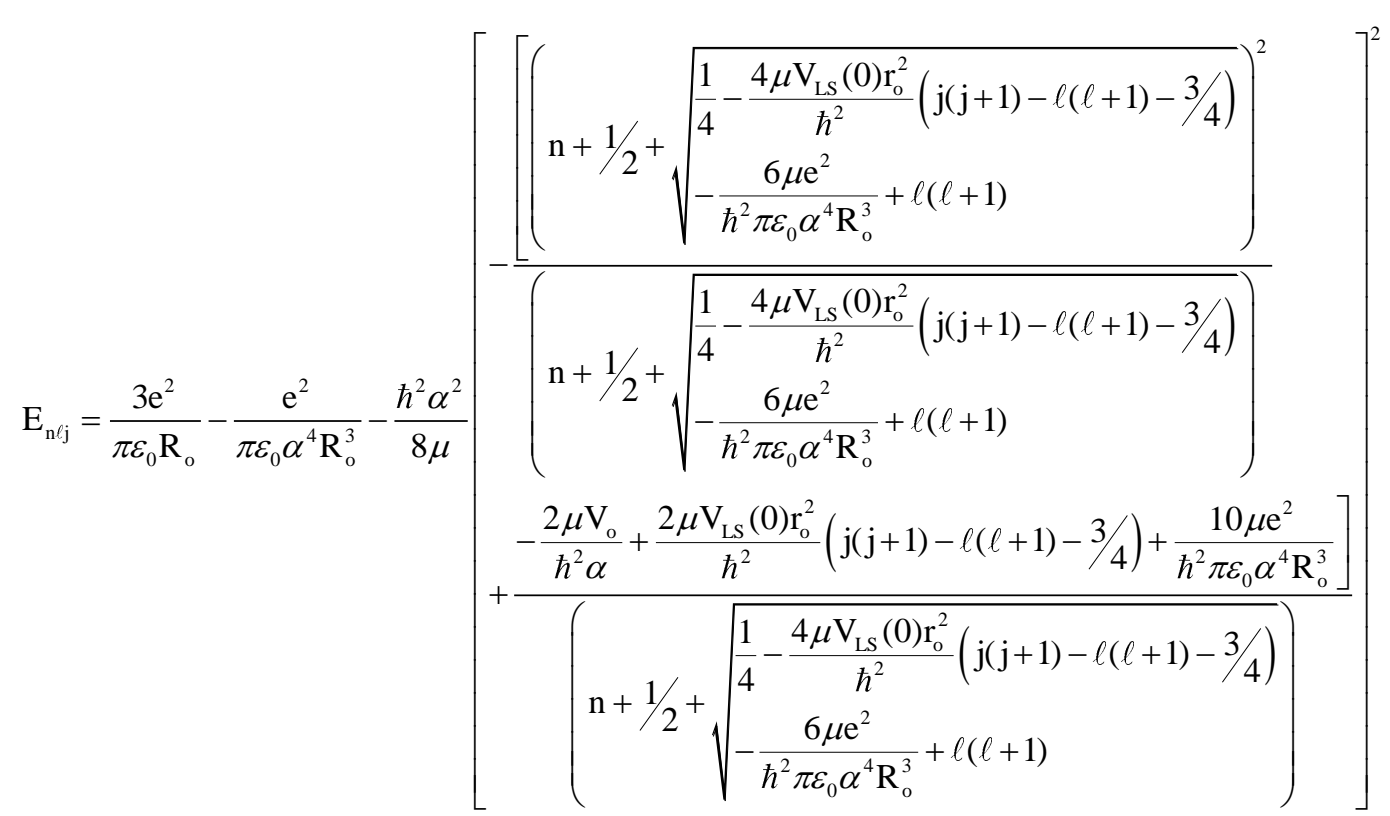

Equation (38) is the energy eigenvalue equation for the proton orbiting the core in a nuclear system. The energy spectrum for a neutron orbit the core in a nuclear system is obtained by setting e in Eq. (38) to zero as the net charge of the neutron is zero, hence there is no Coulomb interaction between the orbiting neutron and the core. Hence the energy eigenvalues for the neutron is given as:

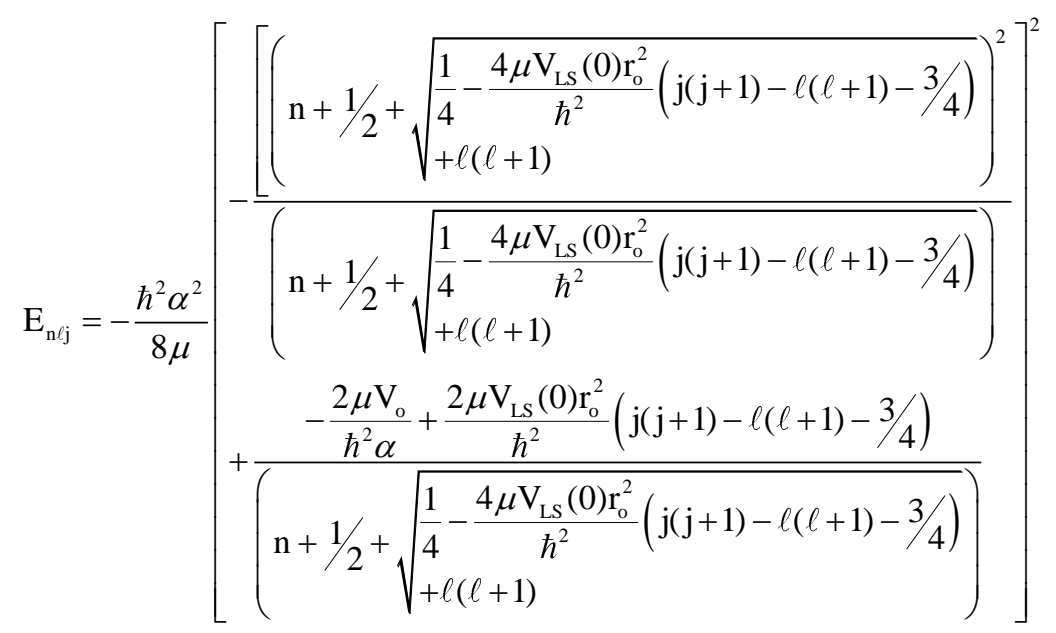

Next we obtain the corresponding single particle eigenfunction as stated in Eq. (8), using the weighted factor $\rho(z)$ which in the form:

$$
\rho(\mathrm{z})=\mathrm{z}^{2 \sqrt{\mathbb{Q}}} \cdot(1-\mathrm{z})^{2 \sqrt{\gamma+\Sigma+\mathbb{Q}}}
$$

Substituting Eq. (40) into Eq. (7), the Rodrigues relation is obtained as:

$$
\begin{aligned}
& \mathrm{y}_{\mathrm{n}}(\mathrm{z})=\mathrm{B}_{\mathrm{n}} \mathrm{z}^{-2 \sqrt{\mathbb{Q}}}(1-\mathrm{z})^{-2 \sqrt{\gamma+\Sigma+\mathbb{Q}}} \frac{\mathrm{d}^{\mathrm{n}}}{\mathrm{dz}^{\mathrm{n}}}\left[\mathrm{z}^{\mathrm{n}+2 \sqrt{\mathbb{Q}}}(1-\mathrm{z})^{\mathrm{n}+2 \sqrt{\gamma+\Sigma+\mathbb{Q}}}\right] \\
& \equiv \mathrm{P}_{\mathrm{n}}^{(2 \sqrt{\mathbb{Q}}, 2 \sqrt{\gamma+\Sigma+\mathbb{Q}})}(1-2 \mathrm{z})
\end{aligned}
$$

where $P_{n}$ is the Jacobi polynomial. Using Eq. (36) and Eq. (41) the wave function is given as:

$$
\psi(\mathrm{z})=\mathrm{N}_{\mathrm{n} \ell} \mathrm{z}^{\sqrt{\mathbb{Q}}}(1-\mathrm{z})^{(1 / 2+\sqrt{\gamma+\Sigma+\mathbb{Q}})} \mathrm{P}_{\mathrm{n}}^{(2 \sqrt{\mathbb{Q}}, 2 \sqrt{\gamma+\Sigma+\mathbb{Q}})}(1-2 \mathrm{z})
$$

where $\mathrm{N}_{\mathrm{n} \ell}$ is the normalization constant. Applying the normalization condition the Normalization constant is obtained by applying the normalization condition as thus: 


$$
\frac{\mathrm{N}_{\mathrm{n} \ell}^{2}}{\alpha} \int_{-1}^{1}\left(\frac{1-\mathrm{y}}{2}\right)^{\omega}\left(\frac{1+\mathrm{y}}{2}\right)^{\mathrm{b}}\left[\mathrm{P}_{\mathrm{n}}^{(\omega, \mathrm{b}-1)} \mathrm{y}\right]^{2} \mathrm{dy}=1
$$

Equation (43) is derived by setting:

$$
\begin{aligned}
& \mathrm{b}=\frac{1}{2}+\sqrt{\gamma+\Sigma+\mathbb{Q}} \\
& \omega=2 \sqrt{\mathbb{Q}}
\end{aligned}
$$

By comparing Eq. (43) to the standard integral of the form Eq. (37) in the ref. [24], we have:

$$
\int_{-1}^{1}\left(\frac{1-p}{2}\right)^{x}\left(\frac{1+p}{2}\right)^{y}\left[P_{n}^{(2 x, 2 y-1)} y\right]^{2} d p=\frac{2 \Gamma(x+n+1) \Gamma(y+n+1)}{n ! x \Gamma(x+y+n+1)}
$$

We derive the normalization constant as:

$$
N_{n \ell}=\left[\frac{\alpha n ! \omega \Gamma(\omega+b+n+1)}{2 \Gamma(\omega+n+1) \Gamma(b+n+1)}\right]^{1 / 2}
$$

\section{SPECial CASES}

\section{A. Case 1: Coulomb potential}

The energy spectrum for adjusted Coulomb potential only can be obtained from the energy spectrum for SHSC potential by setting some parameters in Eq. (38) to zero. We set the parameters of SHSC to zero; $\mathrm{V}_{\mathrm{o}}$ $=0, \mathrm{~V}_{\mathrm{ls}}=0$.

$$
\left.\mathrm{E}_{\mathrm{n} \ell \mathrm{j}}=\frac{3 \mathrm{e}^{2}}{\pi \varepsilon_{0} \mathrm{R}_{\mathrm{o}}}-\frac{\mathrm{e}^{2}}{\pi \varepsilon_{0} \alpha^{4} \mathrm{R}_{\mathrm{o}}^{3}}-\frac{\hbar^{2} \alpha^{2}}{8 \mu}\left[-\frac{\left[\left(\mathrm{n}+1 / 2+\sqrt{\frac{1}{4}-\frac{6 \mu \mathrm{e}^{2}}{\hbar^{2} \pi \varepsilon_{0} \alpha^{4} \mathrm{R}_{\mathrm{o}}^{3}}+\ell(\ell+1)}\right)^{2}+\frac{10 \mu \mathrm{e}^{2}}{\hbar^{2} \pi \varepsilon_{0} \alpha^{4} \mathrm{R}_{\mathrm{o}}^{3}}\right.}{\left(\mathrm{n}+1 / 2+\sqrt{\frac{1}{4}-\frac{6 \mu \mathrm{e}^{2}}{\hbar^{2} \pi \varepsilon_{0} \alpha^{4} \mathrm{R}_{\mathrm{o}}^{3}}}+\ell(\ell+1)\right.}\right)\right]^{2}
$$

Equation (47) is the energy spectrum for the adjusted Coulomb potential.

\section{B. Case 2: Hulthen potential}

The energy spectrum for Hulthen potential is obtained from the CHSC potential by setting $\mathrm{V}_{\mathrm{ls}}=0$ and $\mathrm{e}$ $=0$;

$$
\mathrm{E}_{\mathrm{n} \ell}=-\frac{\hbar^{2} \alpha^{2}}{8 \mu}\left[\frac{(\mathrm{n}+1+\ell)^{2}-\frac{2 \mu \mathrm{V}_{0}}{\hbar^{2} \alpha}}{(\mathrm{n}+1+\ell)}\right]^{2}
$$

The special cases of our solution to the Schrodinger equation for SHSC potential has proven that the SHSC potential offers a broad base of application. From our solution equation Eq. (38) and Eq. (39) are applicable to nuclear systems, Eq. (47) gives the Coulomb contribution to the nuclear bound state energy of a proton, while (48) can be applicable to solid state and molecular systems.

\section{CONCLUSION}

In this work, we have studied in the solution of Schrodinger wave equation for SHSC potential using the NIkiforov-Uvarov method. Applying necessary approximations the single particle energy spectrum for a nucleus with one nucleon orbiting core with a closed shell. We also considered two special cases of the potential and obtain their respective energy spectra. Although the superposition of Hulthen, spin-orbit and adjusted Coulomb potential which we called SHSC potential is novel with respect to its application to the non-relativistic wave equation, the special cases shows that our results is in agreement with literature. As such, our results for the SHSC potential unarguable is valid within reasonable level of approximation. The corresponding normalized single particle wave function for SHSC has been obtain in this study. 


\section{ACKNOWLEDGMENT}

E. A. Thompson will like to thank E.S. Williams for his encouragement for the successful completion of this work.

\section{REFERENCE}

[1] O. Bayrak, G. Kocak and I. Boztozun. Any 1-state solution of the Hulthen potential by asymptotic iteration method. Journal of physics A: Mathematical and general, 2008: 11521-11529.

[2] H. Hassanabadi, B. Yazarlko, A. N. Ikot, N. Salahi and S. Zarrinkamr. Exact analytical solutions of Schrodinger equation for Hua plus modified Eckart potential. Indian journal of Physics, 2013; 87(12):1219-1223.

[3] S. H. Dong. Exact and proper quantization rules and langer modification wave equation in higher dimensions. Springer, Dordrecht, 2011

[4] S. H. Dong. Factorization method in quantum mechanics, Springer, Dordrecht, 2007.

[5] A. Sinha, R. Roychoudbury and Y. P. Varshni. Shifted 1/N expansion for confined quantum systems. Canadian journal of physics, 2000; 78(2):141-152.

[6] E. S. William, E.P. Inyang, E. A. Thompson. Arbitrary 1-solution of the Schrodinger equation interacting with Hulthen-Hellman potential model. Rev. Mex. Fis. 2020; 66(6):1-11.

[7] D. Agboola. Schrodinger equation with Hulthen potential plus Ring-shape potential. Communication in theoretical physics, 2011; 55(6):972-976.

[8] M. Alberg and L. Wilets. Exact solution to the Schrodinger equation for potential with Coulomb and Harmonic Oscillator terms. Physics letter A, Elsevier, 2001; 286:7-14.

[9] A. Niknam, A. A. Rajabi, and Solaimani. Solution of D-dimensional Schrödinger equation for Woods - Saxon potential with Spin-orbit, Coulomb and Centrifugal terms through a new Hybrid Numerical fitting Nikiforov-Uvarov Method. Journal of theoretical and applied physics. Springer, 2016; 10:53-59.

[10] L. Hulthe'n. U” ber die eigenlo“sunger der Schro"dinger-Gleichung des deuterons. Ark. Mat. Astron. Fys. A 28, $1942 ; 5$.

[11] D. Agboola. The Hulthen Potential in D - dimensions. Physica Scripta 2009; 80, 065304:1-5.

[12] S. M. Ikhdair. Approximate Eigenvalues and Eigenfunction solutions for the Generalised Hulthen Potential with any Angular Momentum, Journal of mathematical chemistry, 2007; 42(3):461-471.

[13] C. O. Edet and P. O. Okoi. Any 1 State solution of the Schrödinger equation for q-Deformed Hulthen plus Generalized Inverse Quadratic Yukawa Potential in Arbitrary Dimensions. Revisita Mexicana de Fisica, 2019; 65:333-344.

[14] M. Mousavi and M. R. Shojaei. Calculation of Energy and Charge Radius for Doubly Magic Nuclei of 41Ca and 41Sc with Extra Nucleon. Chinese Journal of physics. Elsevier, 2016; 1-6.

[15] M. Mousavi, and M. R. Shojaei. Bound State Energy of Double Magic Number plus one Nuclei with Relativistic Mean Field Approach. Pramana Journal of Physics, 2017; 88(21):1-5.

[16] T. Otsuks, R, Fujimoto, Y. Utsuno et al. Magic numbers in Exotic Nuclei and spin-isospin properties of the NN interaction. Phys. Rev. let., 2001;87(8):1-4.

[17] D. Amos and I. Talmi. Nuclear shell model theory, Academic press Inc., New York, 1965.

[18] M. R. Pahlavani and S. A. Alavi. Solution of Woods-Saxon potential with spin-orbit and centrifugal terms through NikiforovUvarov method. Comm. Theor. Phys., 2012; 58(5):739-743.

[19] A. F. Nikiforov and V.B. Uvarov. Special function of mathematical physics. Birkhauser, basel, 1988.

[20] R. L. Greene and C. Aldrich,Variational wave functions for a screened Coulomb potential. Phys. Rev. A., 1976;14:2363.

[21] S. H. Dong, W. C. Qiang, G. H. Suin and V. R. Bezerra. Analytical approximation to the 1-wave solution of the Schrodinger equation with the Eckart potential. J. phys. A. 2007; 40:1-7.

[22] C. A. Ebomwonyi, M. C. Onate, M. C. Onyeaju and A. N. Ikot. Any 1 state solution of the Schrodinger equation interacting with Hellmann generalized Morse potential model. Kaibala Intl. J. Mod. Sc., 2017; 3:1-10.

[23] A. Tas, O. Aydogdu, and M. Sati. Relativistic spinless particle with position dependent mass: Bound state and Scattering phase shift. J. Korean Phys. Soci. 2017; 70:896-904.

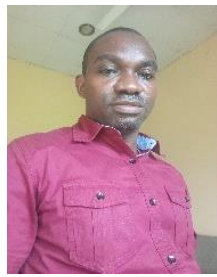

E. A. Thompson was born in Akwa Ibom State on the 27th July. He holds a Ph. D in Theoretical Physics, M.Sc. in Nuclear Physics, and B.Sc. in Physics all from the University of Calabar, Calabar, Cross River State, Nigeria.

He is currently a lecturer in the University of Calabar, Calabar, Nigeria. He has published in reputable journals of Physics. 